\section{DENTISTS AND DOCTORS MUST COLLABORATE ON OSTEOPOROSIS CARE, US STUDY URGES}

Dentists and doctors must collaborate to improve early detection and treatment of patients with osteoporosis, particularly regarding bisphosphonates, according to a review by two US researchers in the Journal of the American Dental Association.

Beatrice J. Edwards, Associate Professor of Medicine at Northwestern University, Chicago, and Cesar A. Migliorati, Professor of Oral Medicine at Nova Southeastern University, Fort Lauderdale, who reviewed randomised controlled trials, say 'Osteoporosis and related fractures are more common than coronary disease, stroke and breast cancer.' Increasingly prevalent, the condition often goes unnoticed until fractures occur. Health costs associated with the disease may triple by 2020 as the population ages, the researchers suggest.

'The prevalence of osteoporosis is increasing rapidly, and fractures associated with this disease can have devastating consequences. It is recommended that physicians and dentists collaborate to improve early detection,' say the authors.

'Oral health maintenance is important in patients with osteoporosis. They often have significant dental needs and their poor oral health can compro- mise systemic health,' the authors say. 'Bisphosphonate therapy or other treatment should be discontinued only after consultation with the patient's physician,' they recommend.

While it has been suggested that patients taking bisphosphonates should be given a 'drug holiday' when undergoing dental treatment involving bone manipulation, "the literature provides no scientific evidence to support the idea that discontinuation of bisphosphonate therapy will improve treatment outcomes,' the authors note.

'Before discontinuing bisphosphonate therapy, dentists and physicians must collaborate to determine the best way to manage the treatment of each patient,' they urge. Consultation with a doctor would allow evaluation of bone mineral density and the risk of fractures, the study notes.

The study 'Osteoporosis and its implication for dental patients' ( $\mathrm{J}$ Am Dent Assoc 2008; 139: 545-552) says it is important to identify patients who are taking bisphosophates by careful history taking. Because these medications are taken weekly or monthly, patients frequently forget to tell their dentists they are on them, it points out.

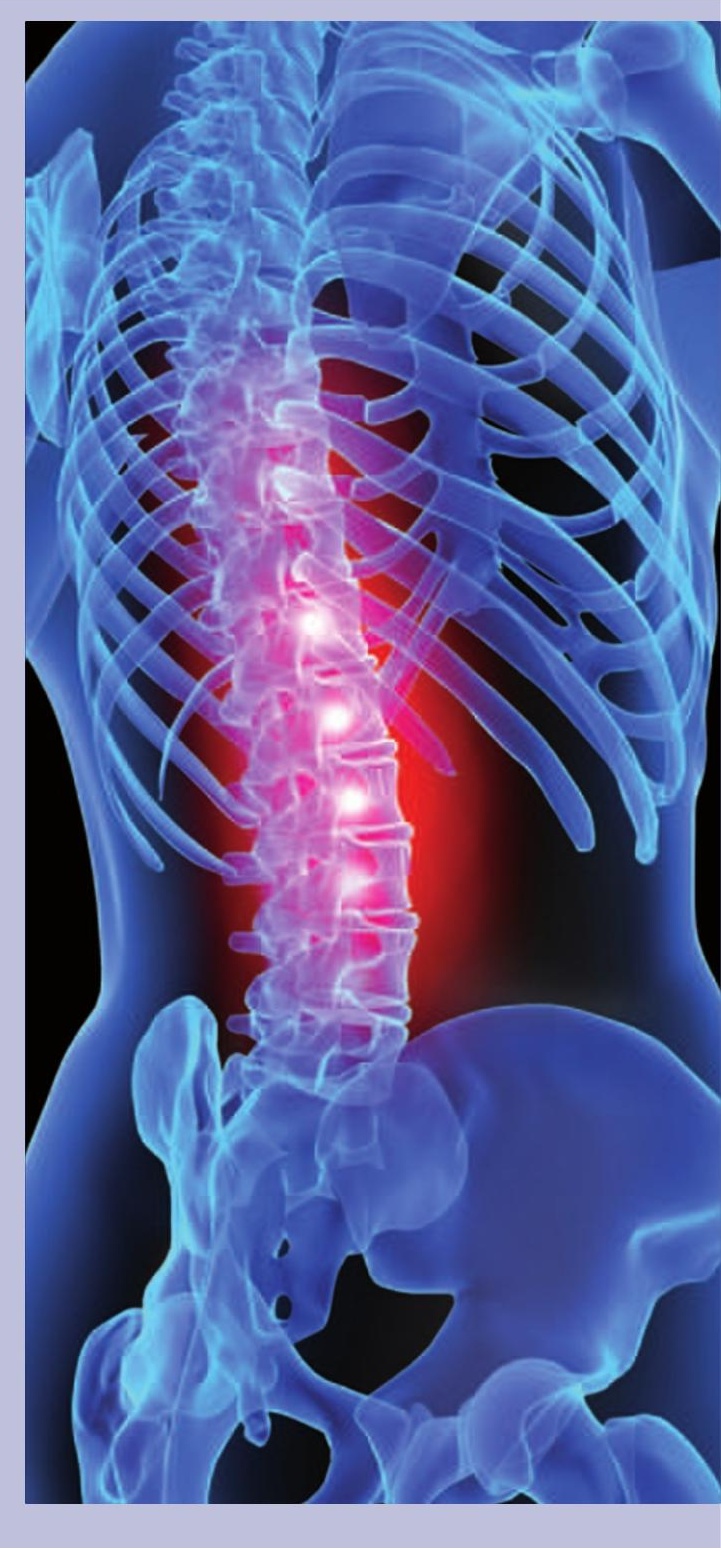

\section{GUM DISEASE ASSOCIATED WITH INCREASED CANCER RISK}

Men with a history of gum disease are at increased risk of developing cancer, according to a follow up study of health professionals in The Lancet Oncology.

The study, led by Dominique Michaud, Reader in Cancer Epidemiology at Imperial College, London, used data from the US Health Professionals FollowUp study which was started in 1986 at Havard. The cohort comprised 48,375 men, including 29,683 dentists.

Follow up questionnaires were sent to all living participants every two years. Baseline gum disease with bone loss, number of natural teeth and tooth loss in the previous two years were recorded, with smoking history and food intake. The median follow up in the study was 17.7 years.
After adjustment for known risk factors, including detailed smoking history and dietary factors, participants with a history of gum disease had a 14 per cent higher risk of cancer compared with those with no history of gum disease. The most common cancers were colorectal, melanoma of the skin, lung, bladder and advanced prostate cancer.

'Periodontal disease might be a marker of a susceptible immune system or might directly affect cancer risk,' the authors conclude. The full paper, 'Periodontal disease, tooth loss, and cancer risk in male health professionals: a prospective cohort study' appears in Lancet Oncology volume 9, pages 550-558.

\section{HEALTH SUPPORT LINE}

Dentists coping with substance abuse can now get directly through to a support scheme by telephoning 02072244671.

The BDA has arranged for calls to this number to be put through to the co-ordinator of the Dentists' Help Support Programme. The programme was established in 1986 to provide confidential help for dentists struggling with drink or drug issues.

The National Clinical Assessment Service (NCAS) is planning to launch a new service to support sick dentists in London in the autumn. An announcement on who will run the service is expected shortly. 